\title{
Droit à l'instruction et respect des convictions religieuses au sens de la CEDH : développements récents
}

\section{Gérard Gonzalez}

\section{OpenEdition}

\section{Journals}

Édition électronique

URL : https://journals.openedition.org/rdr/1470

DOI : $10.4000 /$ rdr. 1470

ISSN : 2534-7462

Éditeur

Presses universitaires de Strasbourg

\section{Édition imprimée}

Date de publication : 6 mai 2021

Pagination : 193-198

ISBN : 979-10-344-0089-8

ISSN : 2493-8637

\section{Référence électronique}

Gérard Gonzalez, « Droit à l'instruction et respect des convictions religieuses au sens de la CEDH :

développements récents ", Revue du droit des religions [En ligne], 11 | 2021, mis en ligne le 06 mai 2021, consulté le 03 mai 2022. URL : http://journals.openedition.org/rdr/1470 ; DOI : https://doi.org/ $10.4000 /$ rdr. 1470 


\section{Droit à linstruction et respect des convictions religieuses au sens de la CEDH: développements récents}

\section{Gérard GONZALEZ}

Université de Montpellier, Institut de droit européen des droits de l'homme (IDEDH)

Université de Strasbourg / CNRS, Droit, Religion, Entreprise et Société (DRES)

$\mathrm{L}$

'article $2 \mathrm{du}$ premier protocole (ci-après $2 \mathrm{P} 1$ ) de la Convention europenne des droits de l'homme prévoit que «nul ne peut se voir refuser le droit à l'instruction» et que «l'État, dans le domaine de l'instruction et de l'enseignement, respectera le droit des parents d'assurer cette éducation et cet enseignement conformément à leurs convictions religieuses et philosophiques». De longues discussions furent nécessaires avant d'inscrire ce droit, absent du texte adopté en 1950 faute d'un consensus assez large, dans le protocole adopté le 20 mars 1952. Les travaux préparatoires révèlent que le prix que beaucoup attachaient à la liberté d'enseignement concrétisée par la création et le soutien à des écoles privées constituait la pierre d'achoppement sur laquelle butèrent les négociations pendant plus de trois ans. Ces débats ne sont pas pour rien dans la formulation négative de la première phrase ( «nul ne peut se voir refuser...») qui tranche avec d'autres, plus précises comme, par exemple, la garantie offerte par la Charte des droits fondamentaux (art. 14) qui préfère une formulation positive du droit à l'instruction. Mais la jurisprudence de la Cour de Strasbourg a éclairci et dynamisé le sens des deux phrases de l'article $2 \mathrm{P} 1^{1}$,

1. V. G. Gonzalez, «Le droit à l'instruction au sens de la Convention européenne des droits de l'homme», RFDA 2010, p. 1003-1010; L.-M. LE RouzIc, Le droit à l'instruction dans la jurisprudence de la Cour EDH, Paris, L'Harmattan, 2015. 
par exemple récemment concernant les discriminations dans le droit à l'instruction de personnes souffrant de handicap ${ }^{2}$ ou encore sur la «diligence et la célérité » imposées aux autorités chargées de la santé publique pour lever dès que possible, une fois tout danger écarté, les mesures restrictives prises pour lutter contre une maladie infectieuse, ce qui inclut les mesures d'absence obligée de l'école des élèves atteints ${ }^{3}$. Deux catégories d'affaires retiennent l'attention et ne sont pas sans échos dans la malheureuse actualité hexagonale de la fin de l'année 2020. La première concerne le champ restreint de l'objection de conscience en milieu scolaire; la deuxième a trait à la restriction d'accès à l'enseignement à domicile.

\section{LE CHAMP RESTREINT DE L'OBJECTION DE CONSCIENCE EN MILIEU SCOLAIRE}

Cette objection devrait pouvoir s'exprimer sur le fondement de la deuxième phrase de l'article 2P1 pour que l'élève soit dispensé d'assister à certains cours ou de participer à certaines activités.

La seconde phrase de l'article 2P1 n'interdit pas à l'État «de répandre par l'enseignement ou l'éducation des informations ou connaissances ayant, directement ou non, un caractère religieux ou philosophique» et ne donne aucun droit aux parents de s'opposer à de tels enseignements. En revanche, le contenu de tels enseignements doit être diffusé «de manière objective, critique et pluraliste» et sans poursuivre «un but d'endoctrinement ${ }^{4}$. L'application de ces principes pose essentiellement le problème de cours «orientés». Jusqu'ici aucune requête de parents en désaccord avec l'assistance obligatoire à des cours d'éducation sexuelle relevant de la biologie ${ }^{5}$ ou dispensés dans une école primaire ${ }^{6}$, de morale non confessionnelle ${ }^{7}$ ou de

2. CEDH, 30 janv. 2018, Enver Şahin c. Turquie: aménagement des locaux universitaires; 10 sept. 2020, C.L. c. Italie: soutien scolaire d'élèves autistes; a contrario déc. 24 janv. 2019, Dupin c. France: scolarisation justifiée en milieu spécialisé d'un enfant autiste compte tenu de son niveau de handicap.

3. CEDH, 6 oct. 2015, Memlika c. Grèce (une forme de lèpre).

4. CEDH, 7 déc. 1976, Kjeldsen, Busk, Madsen et Pedersen c. Danemark, § 53; CEDH, Gde ch., 29 juin 2007, Folgero et a. c. Norvège, \& 84.

5. Affaire Kjeldsen précit. ; CEDH, déc. 25 mai 2000, Jimenez Alonso et Jimenez Merino c. Espagne.

6. Déc. 19 déc. 2017, A.R. et L.R. C. Suisse.

7. Comm. EDH, déc. 9 sept. 1992, Sluijs c. Belgique; déc. 8 sept. 1993, Bernard et a. c. Luxembourg. 
cours d'éthique ${ }^{8}$ n'a débouché sur un constat de violation de la Convention. Il en va autrement des cours axés sur la religion ${ }^{9}$. Si la Cour a reconnu la liberté de rendre de tels cours obligatoires, c'est sous réserve de prévoir un régime de dispense très souple seul à même d'assurer le respect des dispositions de la deuxième phrase de l'article 2P1 lue à la lumière de l'article 9 de la Convention garantissant la liberté de pensée, de conscience et de religion. L'arrêt Papageorgiou et a. c. Grèce du 31 octobre 2019 soulève l'obligation faite à des parents souhaitant dispenser leur enfant du cours de religion, obligatoire en Grèce pour tout élève du primaire comme du secondaire, de déclarer solennellement qu'ils ne sont pas chrétiens orthodoxes (§ 81). Les parents ou les élèves ne sont donc pas tenus de révéler la nature de leurs convictions ${ }^{10}$ mais de donner une forme de publicité à celle qu'ils ne partagent pas (§ 85). De plus, l'administration scolaire doit contrôler la réalité de la «déclaration solennelle» au regard notamment des certificats de naissance indiquant la religion des parents et toute contradiction entre les deux peut faire l'objet de poursuites. Le caractère très intrusif de ce mécanisme le disqualifie au regard de la deuxième phrase de l'article 2P1 interprétée à la lumière de l'article 9. Il est donc inconventionnel d'obliger les parents ou les enfants à dévoiler cet aspect intime de leur vie privée que constituent leurs convictions religieuses ou leur absence. Tout est ici affaire de nuance, de respect et de tolérance, chacun étant invité à faire la part entre ses droits et ses devoirs.

Dans l'affaire Perovy c. Russie (20 oct. 2020) les parents d'un enfant de sept ans se prétendent sans succès victimes d'une violation de l'article 2P1 au motif que leurs fils a dû assister, le jour de la rentrée, à une cérémonie de bénédiction de sa classe selon le rite orthodoxe russe alors qu'ils appartiennent à une autre confession. Leur fils également requérant sur le fondement de l'article 9 de la Convention n'eut pas plus de réussite. La Cour juge en effet, à une courte majorité (4 voix contre 3 ), que, «bien que regrettable», il s'agit d'un «incident isolé dans l'éducation [de l'enfant], de portée et de durée limitée» (§ 66) et les autorités nationales ont agi «rapidement et de façon adéquate» à la plainte des parents (§ 68). La Cour semble confirmer qu'elle est peu réceptive aux plaintes de parents ou d'enfants dont la conscience religieuse ou philosophique est ponctuellement heurtée par l'obligation de participer à une activité contraire à leurs convictions. Déjà, dans l'affaire Valsamis c. Grèce (27 nov. 1996) bien qu'elle «s'étonne qu'il puisse être

\footnotetext{
8. CEDH, déc. 6 oct. 2009, Appel-Irrgang c. Allemagne.

9. Affaire Folgero, précit. ; CEDH, 9 oct. 2007, Zengin c. Turquie.

10. Comme dans Folgero précit., § 98; Zengin précit., § 73.
} 
exigé des élèves, sous peine de renvoi scolaire même limité à une journée, de défiler en dehors de l'enceinte scolaire un jour férié», elle a pu juger qu'une telle sanction infligée à une élève témoin de Jéhovah et professant des croyances pacifistes, ne violait pas la Convention (2P1 et 9) malgré la présence de militaires au premier plan de cet évènement. Elle admet également, dans l'enceinte scolaire, une atteinte plus durable aux droits revendiqués par des parents ou des élèves dès lors qu'un intérêt supérieur areligieux dicte une ingérence dans la liberté des élèves de manifester leur religion ${ }^{11}$ ou que, manifestant l'attachement de l'État à la religion majoritaire, un symbole «passif» de cette religion est accroché dans les salles de classe ${ }^{12}$. Si l'arbitrage de la Cour dans ces dernières situations de conflits de droits trouvant place dans l'enceinte proprement dite de l'école peut convaincre, l'arrêt Valsamis choque par le dédain affiché à l'égard de convictions fortes bafouées en dehors de cette enceinte et, de surcroît, du temps scolaire proprement dit, situation amenée à se répéter d'année en année, ce qui est le principe d'une commémoration. Si cet arrêt ne grandit pas la Cour, peut-on en dire autant de l'arrêt Perovy? Malgré l'opinion fortement dissidente de trois des sept juges, il ne semble pas. L'élément déterminant à notre sens réside dans la réaction disciplinaire des autorités nationales à l'égard de l'enseignant et du principal de l'établissement et l'assurance que ce type d'incident ponctuel «ne se reproduirait plus» (§ 68). Le seul fondement de la requête demeurait l'insuccès de la procédure judiciaire intentée par les intéressés pour dommage moral contre l'école. L'échec de cette démarche procédurière n'a pas ému la Cour. On peut l'admettre malgré la «dimension particulièrement active » du rite et son impact possible sur un enfant de sept ans habitué à d'autres cérémonies comme le soulignent les juges dissidents.

\section{L'ENSEIGNEMENT À DOMICILE}

Cette question, redevenue d'actualité avec le débat sur les «séparatismes », était au cœur de l'arrêt Wunderlich c. Allemagne (CEDH, 10 janv. 2019). Cette affaire concerne l'atteinte au droit au respect de la vie privée et familiale (art. 8) que constituerait la privation de certains aspects de l'autorité parentale et le retrait pendant trois semaines de leurs quatre enfants à leurs

11. L'interdiction de signes religieux dans les écoles, voire les universités, au nom de la défense de la laïcité: CEDH, Gde ch., 10 nov. 2005, Leyla Sahin c. Turquie; CEDH, 4 déc. 2008, Dogru c. France et Kervanci c. France; déc. 30 juin 2009, Aktas, Bayrak, Ghazal, Jasvir Singh et Ranjit Singh c. France: AJDA 2009, p. 2077, note G. GonZalez.

12. CEDH, Gde ch., 18 mars 2011, Lautsi c. Italie. 
parents après que ceux-ci eurent obstinément refusé de les envoyer à l'école pour des motifs que l'arrêt ne précise pas. La Cour a déjà été confrontée à cette question, au regard notamment d'un refus de scolarisation sur un fondement religieux. Elle a déclaré irrecevable, en 2006, la requête de parents souhaitant éduquer leurs enfants en accord avec la Bible et les valeurs chrétiennes mais n'ayant pas obtenu la permission requise en Allemagne d'éduquer leurs jeunes enfants à domicile plutôt qu'à l'école primaire (déc. 11 sept. 2006, $\mathrm{n}^{\circ}$ 35504/03, Konrad et a. c. Allemagne). Pour déclarer la requête manifestement mal fondée, la Cour avait retenu que l'école transmet non seulement la connaissance mais favorise aussi «l'intégration et les premières expériences de la société » et les parents demeurent libres d'éduquer leurs enfants en dehors des heures scolaires et le week-end. Elle approuvait aussi le raisonnement de la Cour constitutionnelle qui «a souligné l'intérêt général de la société à éviter l'émergence de sociétés parallèles fondées sur des convictions philosophiques distinctes et l'importance d'intégrer les minorités dans la société». On notera que de «l'émergence de sociétés parallèles» au «séparatisme» il n'y a qu'un pas! Dans l'affaire Wunderlich la Cour donne plus de solennité à sa position en adoptant cette fois un arrêt de chambre à l'unanimité à l'égard d'une requête mettant en cause la grave sanction de privation de certains aspects de l'autorité parentale et de retrait des quatre enfants à leurs parents durant trois semaines ${ }^{13}$. Elle confirme la validité du système de scolarisation obligatoire en Allemagne sauf dérogation dûment autorisée tant au regard de l'article 8 que de l'article $2 \mathrm{P} 1^{14}$. Ce qui est en cause ici c'est donc la nécessité de la sanction prise au regard du droit à une vie familiale normale dans le cadre de cette restriction conventionnelle au droit des parents de privilégier l'instruction à domicile pour des raisons qui, dans cette affaire, ne sont pas mentionnées. Prévue par la loi, la mesure adoptée poursuit les buts légitimes de «protection de la santé et de la morale» et des «droits d'autrui» (§ 45). Le contrôle de proportionnalité de l'ingérence est effectué à l'aune de «l'intérêt supérieur de l'enfant» qui, selon les cas, peut l'emporter sur celui des parents (§ 46). La Cour souligne l'ample marge d'appréciation des États lorsqu'il s'agit de prendre soin d'enfants, de leur prise en charge (§ 47). Elle réitère son affirmation de la décision Konrad concernant la nécessité d'intégrer

13. Le fondement de l'article 8 semble le plus pertinent. En effet celui de l'article 2P1 est contre-productif puisque sa deuxième phrase garantit justement le droit des parents d'instruire leurs enfants selon leurs convictions et interdit tout prosélytisme d'État. Quant à l'article 9, il apparaît clairement que le fondement de la liberté de religion ne peut justifier à lui seul le refus de scolarisation au profit d'un enseignement à domicile.

14. Konrad précit.; CEDH, déc. 13 sept. 2011, Dojan et a. C. Allemagne: refus d'assistance à certains cours obligatoires; Comm. EDH, déc. 9 juillet 1992, Leuffen c. Allemagne. 
les enfants dans la société et d'éviter «l'émergence de sociétés parallèles», considérations qui cadrent avec l'importance du pluralisme dans toute société démocratique (§ 50). Elle juge «que l'application de l'obligation de fréquentation scolaire, pour prévenir l'isolement social des enfants des requérants et assurer leur intégration dans la société, était une raison pertinente pour justifier le retrait partiel de l'autorité parentale» et «que les autorités nationales ont raisonnablement présumé, sur la base des informations dont elles disposaient, que les enfants étaient menacés par les demandeurs en ne les envoyant pas à l'école et en les maintenant dans un système familial "symbiotique" » (§ 51). Un point néanmoins retient l'attention. La chronologie nationale s'est étalée sur plusieurs années. De 2005, date à laquelle l'une des filles des requérants était en âge d'être scolarisée, jusqu'en août 2013, date des mesures contestées, les enfants ne furent pas scolarisés. Mais une évaluation effectuée en septembre 2013 démontra que «le niveau de connaissance des enfants n'était pas alarmant et qu'ils n'étaient pas retenus de fréquenter l'école contre leur volonté» (§ 23). En conséquence le juge national décida que le retrait permanent des enfants à leurs parents ne constituait plus une mesure proportionnée compte tenu du plus grand impact qu'aurait cette mesure sur les enfants par rapport à la scolarisation à domicile par leurs parents (§ 23). Ce constat interroge. La Cour disqualifie cet argument en relevant que les résultats de l'évaluation n'étaient pas connus au moment où les services de l'enfance prirent les mesures contestées et rappelle que «même des jugements ou évaluations erronés de professionnels ne rendent pas en soi les mesures de garde d'enfants incompatibles avec les exigences de l'article $8 »$ (§ 52). $\mathrm{Au}$ vu des éléments en leur possession, les décisions prises par ces services apparaissent proportionnées et aucune violation de l'article 8 n'est constatée. La Cour renforce ainsi le poids du principe de précaution qui doit guider les services ayant en charge les intérêts des enfants ${ }^{15}$. En conclusion, du point de vue de la Convention européenne telle qu'interprétée par la Cour, l'enseignement prodigué à domicile en lieu et place d'une scolarisation n'est, en soi, ni un droit ni une liberté comme on a pu l'entendre au lendemain du discours du Président français préconisant, comme moyen de lutte contre les «séparatismes», l'interdiction de l'enseignement à domicile sauf pour cas de santé. L'intérêt supérieur de l'enfant passe, pour la Cour européenne, par sa socialisation et celle-ci s'enracine dans la scolarisation obligatoire des enfants. Les États conservent dans ce domaine les coudées franches.

15. Elle condamne les États dont le système de protection de l'enfance en danger a failli: par ex., CEDH, 4 juin 2020, Association Innocence en danger et association Enfance et Partage c. France: violation par l'État défaillant de l'article 3 de la Convention (traitements inhumains et dégradants). 